\title{
LuxS impacts on LytA-dependent autolysis and on competence in Streptococcus pneumoniae
}

\begin{abstract}
Correspondence
Marie-Claude Trombe

trombe@cict.fr
\end{abstract}

Received 4 August 2005

Revised 1 November 2005

Accepted 7 November 2005

\author{
Susana Romao, ${ }^{1}$ Guido Memmi, ${ }^{2}$ Marco R. Oggioni ${ }^{2,3}$ \\ and Marie-Claude Trombe ${ }^{1}$
}

\author{
${ }^{1}$ Interactions et Signalisation Cellulaires: Relation Hôte-Pathogène, EA3036, IFR31, CHU \\ Rangueil, Université Paul Sabatier, 31403 Toulouse, France \\ ${ }^{2}$ Laboratorio di Microbiologia Molecolare e Biotecnologia, Dipartimento di Biologia Molecolare, \\ Università di Siena, Siena, Italy \\ ${ }^{3}$ UOC Batteriologia, Azienda Ospedaliera Universitaria Senese, Siena, Italy
}

The ubiquitous protein LuxS with S-ribosylhomocysteinase activity is involved in S-adenosyl methionine detoxification, $\mathrm{C}-1$ unit recycling and the production of autoinducers that allow the cell to sense and respond to cell density. Independent reports describe the impact of LuxS deficiency on Streptococcus pneumoniae virulence in the mouse. In vitro, LuxS deficiency confers discrete phenotypes. A combined approach using genetic dissection and mixed-culture experiments allowed the involvement of LuxS in the developmental physiology of S. pneumoniae to be investigated. Functional LuxS was found to be related on the one hand to down-regulation of competence, and on the other hand to attenuation of autolysis in cultures entering stationary phase. The competence phenotype of luxS mutant bacteria was complemented by media conditioned by competence-defective ComABO bacteria, but not by BSA. The autolytic phenotype was complemented by BSA, but not by conditioned supernatants. It is suggested that the impact of LuxS on competence, but not on autolysis, involves cell-cell communication. The phenotype of luxS mutant strains reveals a hierarchy in the competence regulatory networks of $S$. pneumoniae.

\section{INTRODUCTION}

LuxS, the S-ribosylhomocysteinase involved in furanosyl borate diester, autoinducer 2 (AI-2) production from $S$ ribosylhomocysteine, is ubiquitous in prokaryotes (Sun et al., 2004). Since its discovery (Bassler et al., 1997) and chemical characterization (Chen et al., 2002), the role of AI-2 in the regulation of light production, biofilm formation and virulence in response to high cell density (quorum sensing) has been elucidated in several Gram-negative bacteria, including Vibrio species (Surette et al., 1999; Miller et al., 2002; Hammer \& Bassler, 2003; Henke \& Bassler, 2004; Vendeville et al., 2005). In some species, furanone uptake and processing involving the $\operatorname{sr} A C D B F G E$ operon has been described (Taga et al., 2003; Xavier \& Bassler, 2005). Bioluminescence activation in Vibrio harveyi by conditioned media represents the test for AI-2 production by a given species and supports the paradigm of AI-2-mediated interspecies cell-cell communication culminating in genetic regulation (Taga \& Bassler 2003; Xavier \& Bassler, 2003). Recent studies give credence to the involvement of LuxS in

Abbreviations: Al-2, autoinducer 2; Cam, chloramphenicol; CSP competence-stimulating peptide; Ery, erythromycin; GSOE, gene splicing by overlap extension; Rif, rifampicin; TCST, two-component signal transduction. host-bacterial communication (Maroui \& Sela, 2003; Sperandino et al., 2003). However, in Neisseria meningitis, AI-2 production is not related to the regulation of specific genes in vitro (Schauder et al., 2005; Dove et al., 2003). In Streptococcus pneumoniae, the impact of LuxS loss-offunction mutations on experimental virulence and genetic expression has been reported; however, results concerning production of AI-2, active in the 'vibrio test', by pneumococci, are divergent (Joyce et al. 2004; Stroeher et al., 2003). In order to get further insight into the function of LuxS in these Gram-positive bacteria, we have investigated its role in vitro on the developmental phenotypes classically observed: competence for genetic transformation during exponential growth, and autolysis when the culture reaches stationary phase. Autolysis is mainly dependent on the activity of the muramidase LytA and on cell-wall composition (for reviews, see Severin et al., 1997; Lopez et al., 1997). The development of competence for genetic transformation involves cell-cell communication and signal transduction. The unmodified heptadecapeptide competence-stimulating peptide (CSP) mediates cell-cell communication. CSP targets the two-component signal transduction (TCST) system ComDE, triggering a signalling cascade by phosphoryl transfer, and culminating in the expression of the sigma-like proteins ComX1 and ComX2, and the $\mathrm{ABC}$ transporter 
ComAB. CSP is encoded by the first gene of the comCDE operon. ComC maturation by ComAB leads to CSP production, the accumulation of which in the medium allows the cell to monitor cell density. CSP, the TCST system ComDE, and ComAB form an autoregulatory circuit. Activation of $\operatorname{comX1}$ and $\operatorname{comX} 2$ results in the activation of the competence regulon that contains the genes encoding the DNA-processing machinery (for a review, see Morrison \& Lee, 2000). Expression of this autoregulated response is modulated by antagonistic positive and negative controls on $\operatorname{com} C D E$ involving the serine/threonine kinase StkP (Echenique et al., 2004) and CiaRH (Echenique et al., 2000, 2004; Echenique \& Trombe, 2001), respectively. In addition, metabolic inputs mediated by the NADH oxidase Nox (Auzat et al., 1999; Echenique \& Trombe, 2001) and the global regulator RegR (Chapuy-Regaud et al., 2003), upstream of the signal transduction cascade, are documented. This indicates complex interplay between metabolic and cell-cell regulation, culminating in the ComAB, CSP, ComDE competence autoregulation circuit. The presence of luxS in the genome of $S$. pneumoniae raises the possibility of an additional actor involved in quorum sensing and metabolic regulation. The impact of a LuxS loss-of-function mutation has been assessed during vegetative growth and in competence development. Data showing LuxS involvement in the control of LytA-dependent autolysis and in competence repression have been obtained by genetic dissection and physiological studies. The results reveal new regulatory networks in addition to the well-described StkP and Com$\mathrm{AB}, \mathrm{CSP}$, ComDE ones.

\section{METHODS}

Bacterial strains and growth conditions. Streptococcus pneumoniae RX isogenic derivatives were routinely used (Table 1). Stock cultures were obtained in casein tryptone medium (CAT) and the cultures were stored at $-80{ }^{\circ} \mathrm{C}$ after addition of $12 \%(\mathrm{v} / \mathrm{v})$ glycerol, as described previously (Trombe et al., 1992). For competence development, CAT medium was complemented by BSA $(0 \cdot 4 \%$, w/v) and $\mathrm{CaCl}_{2}(0 \cdot 1 \mathrm{mM})$, and the $\mathrm{pH}$ was adjusted to 8 to make CTM (CAT transformation medium) (Morrison et al., 1984). Antibiotics were used at the following concentrations: erythromycin (Ery), $2 \mathrm{mg} \mathrm{l}^{-1}$; chloramphenicol (Cam), $10 \mathrm{mg} \mathrm{l}^{-1}$; rifampicin (Rif), $2 \mathrm{mg} \mathrm{l}^{-1}$. Conditioned supernatants were from $5 \mathrm{~h}$ cultures in CTM, pH 8. The bacteria were eliminated by two rounds of centrifugation at $5000 \mathrm{~g}$ and $4{ }^{\circ} \mathrm{C}$, and aliquot fractions of the supernatants were kept frozen at $-80{ }^{\circ} \mathrm{C}$.

Construction of IuxS deletion replacement allele. A deletion replacement mutation in $\operatorname{luxS}$ has been obtained by gene splicing by overlap extension (GSOE) (Iannelli \& Pozzi, 2003). In brief, to obtain the luxS knock-out strain, a $761 \mathrm{bp}$ fragment upstream of SP0340 was amplified using primers IF280a1 (ACCTATGGAGAAACCACTGTCAAC) and IF280a2 (TGTCAGATAGGCCTAATGACTGGCTTTTATAATCTTTACTTACGATATAAGCGGTC) (in the latter primer, the $5^{\prime}$ part matches cat and the $3^{\prime}$ part matches the region upstream of $\operatorname{luxS}$ ), and a fragment of $873 \mathrm{bp}$ downstream of SP0340 was amplified with primers IF280c1 (TCACATTATCCATTAAAAATCAAACAAATTTTCATTCTGTCTTAGACTTTCTCACGAA) (in this primer, the $5^{\prime}$ part matches cat and the $3^{\prime}$ part matches the region downstream of $l u x \mathrm{~S}$ ) and IF280c2 (AGACAAGGTGATTGGTCAAGATAAG). The resistance cassette used for the luxS mutant was cat (Claverys et al., 1995), which confers resistance to chloramphenicol. Extension of the overlaps by DNA polymerase yielded a recombinant DNA molecule which included the cassettes between the two pneumococcal DNA fragments. The PCR product was used to transform strain Cp1015.

Construction of $S$. pneumoniae mutant strains. Transformation of $S$. pneumoniae, transformant recovery and verification of the mutations were performed as described in Auzat et al. (1999). In brief, the luxS-mutated allele produced by GSOE was introduced into the Cp1015 genetic background by CSP-induced transformation. The yield of chloramphenicol-resistant transformants increased with DNA concentration, and the kinetics showed a slope of 1 , indicating that a single transformation event led to transformant recovery. DNA from one chloramphenicol transformant was used as

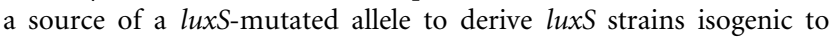
the strains described in Table 1.

Growth measurements. Bacterial growth and autolysis were evaluated by measurement of $\mathrm{OD}_{400}$ of cultures in CAT medium (Trombe et al., 1992) at $37^{\circ} \mathrm{C}$ and at the indicated $\mathrm{pH}$. For complementation studies, BSA fraction $\mathrm{V}$ was used at the indicated concentration. Viability was measured by c.f.u. counting on blood agar plates. The $\mathrm{pH}$ of the medium was measured with the $\mathrm{pH}$ indicator Spezialindikator $6 \cdot 5$ to 10 (Merck).

Competence measurements. Competence development during growth was evaluated in cultures by transformation tests using DNA from strain Cp1016, isogenic to strain Cp1015, but carrying the

Table 1. Recipient strains for the luxS deletion replacement mutation

\begin{tabular}{|c|c|c|c|}
\hline Strains & Relevant genotype & Phenotype & Source or reference \\
\hline Cp1015 & $\mathrm{RX}$ derivative; strl, hexA & $\mathrm{Sm}^{\mathrm{R}}$ & Morrison et al. (1984) \\
\hline Cp1095 & lytA::ery & Ery $^{\mathrm{R}}$ & Trombe et al. (1992) \\
\hline Cp1800 & ciaR: :pPt4 & $\mathrm{Sp}^{\mathrm{R}}$ & Echenique et al. (2000) \\
\hline Cp1008 & comA::ery & Ery $^{\mathrm{R}}$ & Morrison et al. (1984) \\
\hline Cp1890 & ciaR::pPt4, lytA::ery & $\mathrm{Sp}^{\mathrm{R}}, \mathrm{Ery}^{\mathrm{R}}$ & Echenique et al. (2004) \\
\hline Cp9000 & stkP::aphA-3 & $\mathrm{Km}^{\mathrm{R}}$ & Echenique et al. (2004) \\
\hline Cp9090 & stkP::aphA-3, lytA:: ery & $\mathrm{Km}^{\mathrm{R}}$, Ery $^{\mathrm{R}}$ & Echenique et al. (2004) \\
\hline Cp9800 & stkP::aphA-3, ciaR::pPt4 & $\mathrm{Km}^{\mathrm{R}}, \mathrm{Sp}^{\mathrm{R}}$ & Echenique et al. (2004) \\
\hline Cp9890 & stkP::aphA-3, ciaR::pPt4, lytA::ery & $\mathrm{Km}^{\mathrm{R}}, \mathrm{Sp}^{\mathrm{R}}, \mathrm{Ery}^{\mathrm{R}}$ & Echenique et al. (2004) \\
\hline
\end{tabular}


Rif $_{32}$ marker (Auzat et al., 1999). In brief, aliquots from cultures in CTM were incubated in the presence of DNA $\left(1 \mu \mathrm{g} \mathrm{ml}^{-1}\right)$ for $20 \mathrm{~min}$ at $25^{\circ} \mathrm{C}$. Appropriate dilutions were plated, and after $2 \mathrm{~h}$ incubation at $37^{\circ} \mathrm{C}$, a layer of agar medium containing or not containing the selective antibiotic was poured on the plates. Colonies were counted after an overnight incubation at $37^{\circ} \mathrm{C}$. The transformation efficiency of each culture was expressed as the percentage of transformed bacteria in the whole population (percentage transformants). When required, the transformed clones were further streaked on antibiotic plates to check their phenotype. Experiments were performed three times with independent stock cultures.

\section{RESULTS}

\section{The luxS region in the Streptococcus pneumoniae genome}

The Streptococcus pneumoniae genome harbours a single $483 \mathrm{bp}$ gene encoding a protein of 160 amino acids matching the Pfam (Protein Families database; http://www.sanger. ac.uk/Software/Pfam/index.shtml) domain consensus for LuxS (statistical significance $E$ value of $3 \cdot 5 \times 10^{-49}$ ). Among $S$. pneumoniae strains, a single mismatch distinguishes the nucleotide sequences of luxS from strains R6 and TIGR4, and no mutation was observed at the protein level. In the genome, luxS is likely organized as a monocistronic operon located in a region flanked by two IS 1380 sequences, which also contains the dextran glucosidase $\operatorname{dex} B$ gene, the $d c w 3$ operon, encoding division and cell wall proteins (Massidda et al., 1998), clpP, encoding an ATP-dependent protease (Robertson et al., 2002; Kwon et al., 2003) and regR, encoding the repressor of hyaluronidase (Chapuy-Regaud et al., 2003) (Fig. 1). This region is next to the capsule operon cps. RegR, ClpP and OrfD (SP0334 yllC, the first gene of DCW3) are involved in virulence (Chapuy-Regaud et al., 2003; Kwon et al., 2003; Palmen et al., 1999; Robertson et al., 2002). OrfD contains the signature of proteins binding $S$ adenosyl methionine, suggesting that its activity may be related to methionine metabolism (Palmen et al., 1999). LuxS is highly conserved within streptococci and other Gram-positive bacteria; however, the organization of the luxS region is not conserved in the published genomes of related species, such as Streptococcus pyogenes, Streptococcus agalactiae, Streptococcus mutans, Enterococcus faecalis and Lactococcus lactis.

\section{Impact of LuxS on developmental competence}

In order to assess the role of LuxS in S. pneumoniae, a deletion-replacement mutation was generated in vitro and used to transform Cp1015 and its isogenic derivatives (see Table 1 and Methods). The impact of the $\operatorname{luxS}$ mutation on the profile of competence expression was strongly dependent on the genetic background of the bacteria, notably on the nature of their stkP and ciaR alleles. In the $s t k P^{+} c i a r^{+}$ genetic background, a deficiency in LuxS delayed competence expression and shifted the competence peak to lateexponential $4 \mathrm{~h}$ cultures from the exponential $2 \mathrm{~h}$ cultures of the $\operatorname{luxS} S^{+}$strain (Fig. 2A). In the competence downregulated stkP mutant strain, luxS replacement mutation allowed a late competence peak in $4 \mathrm{~h}$ cultures (Fig. 2B). In the ciaRO genetic background, luxS mutation widened the competence window, as shown by competence expression in $1 \mathrm{~h}$ cultures, and consistently in CiaRH- and StkP-deficient strains, luxS mutation further derepressed competence (Fig. 2C, D). These data indicate negative competence control by LuxS in addition to CiaRH. The modest effect of luxS mutation in the wild-type genetic background reveals a hierarchy in the competence regulatory networks, with a dominant role for StkP and CiaRH under the conditions of the study.

\section{LuxS and cell-cell communication}

In order to assess the mechanism of the LuxS control of competence, notably with regard to CSP regulation, mixed cultures from strains $\operatorname{luxS}$ and Cp1008 (comA::ery) (Morrison et al., 1984) in a $1: 1$ ratio were submitted to transformation tests (see Methods). Putative complementation of the defect in CSP production due to the comA:: ery mutation in strain Cp1008 by the com $A^{+} \operatorname{luxS}$ cells, and, reciprocally, repression of late competence in luxS mutant cells by the $\operatorname{luxS^{+}} \operatorname{comA}:$ : ery bacteria, were monitored by transformation tests during growth of the mixed culture. When mixed cultures were considered, they showed a profile

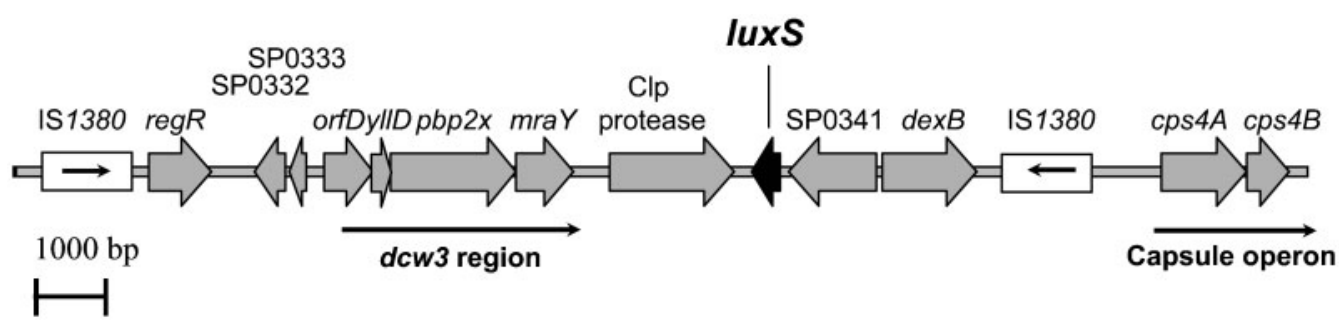

Fig. 1. The luxS locus. Genetic organization of a $22 \mathrm{~kb}$ genomic region of $S$. pneumoniae TIGR4 (GenBank accession AE005672) around luxS SP0340. ORFs are shown as arrows, while insertion sequences are shown as boxes. Hairpin structures indicative of transcriptional terminators are present between regR and SP0332 $\left(\Delta G_{37.0}^{0}=-34.93 \mathrm{kcal} \mathrm{mol}^{-1}\right.$, $\left.146 \cdot 1 \mathrm{~kJ} \mathrm{~mol}^{-1}\right)$, and between SP0341 and luxS $\left(\Delta G_{37.0}^{0}=-11 \cdot 65 \mathrm{kcal} \mathrm{mol}^{-1}, 48 \cdot 74 \mathrm{~kJ} \mathrm{~mol}^{-1}\right)$. 
(A)

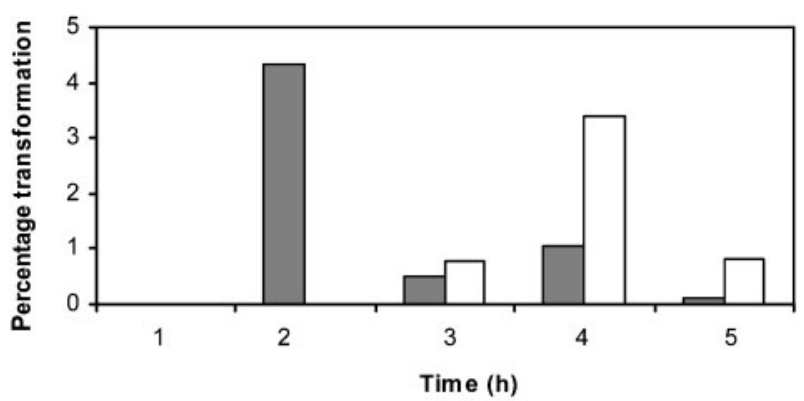

(B)

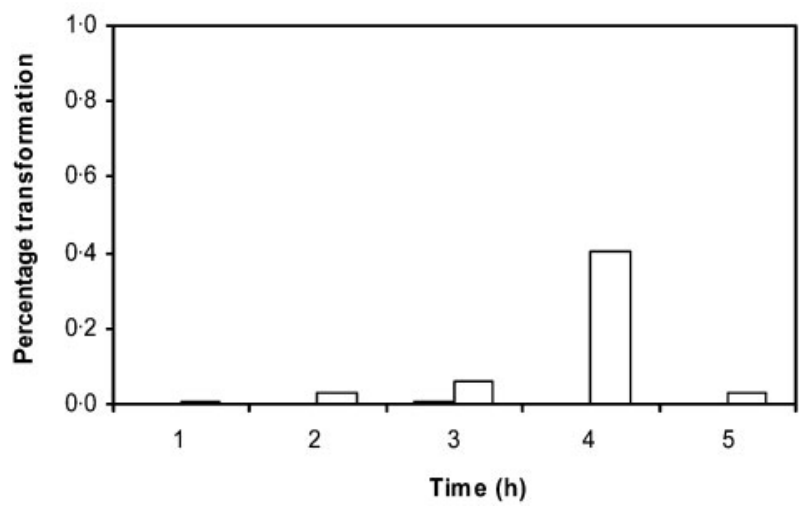

(C)

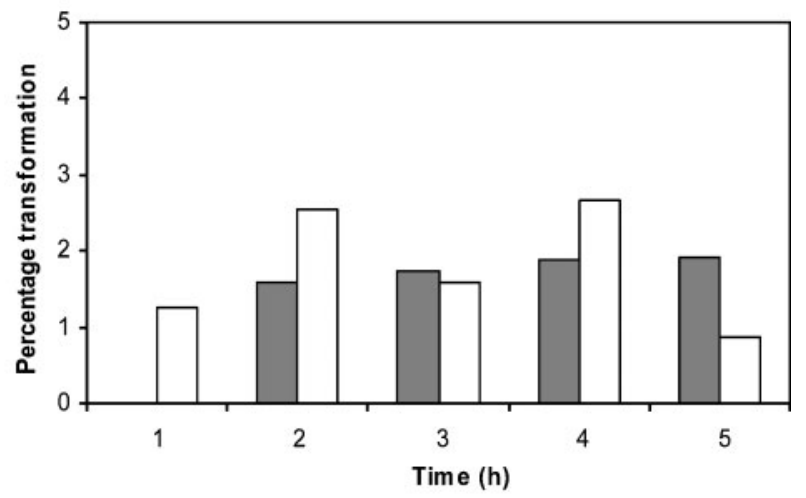

(D)

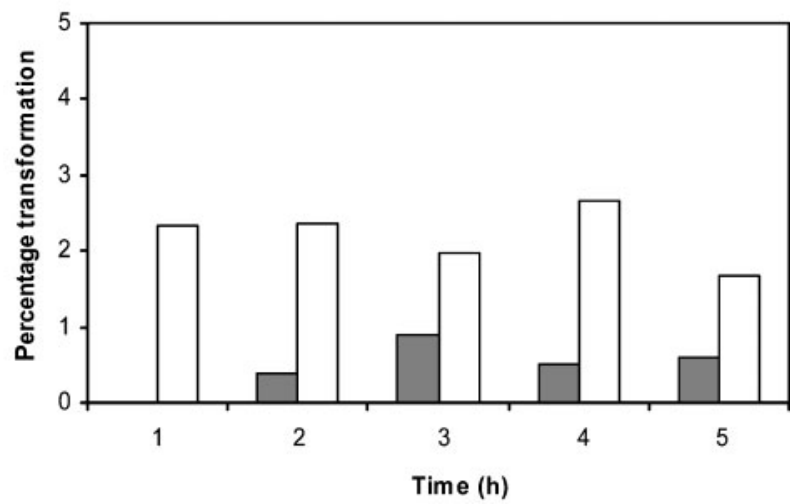

Fig. 2. Impact of luxS mutation on competence development in different genetic backgrounds. Competence development in cultures of (A) the wild-type strain Cp1015 and its luxS isogenic derivative, (B) the stkP mutant strain Cp9090 and its luxS isogenic derivative, (C) the ciaR mutant strain $\mathrm{Cp} 1800$ and its luxS isogenic derivative, and (D) the ciaR stkP strain Cp1890 and its isogenic luxS derivative. Transformation tests (see Methods) were performed on cultures and the percentage recovery of transformants (Methods) in the whole population, taken as representative of the competence level of a given culture, was calculated. The mean value of the data obtained in three independent experiments and differing by less than $10 \%$ is given. Grey bars, lux ${ }^{+}$strains; white bars, lux $S^{-}$strains.

of RifR transformant recovery comparable to that of the wild-type strain. No transformants were recovered during late-exponential growth (Fig. 3 and insert). Twelve transformed clones, obtained from the $2 \mathrm{~h}$ culture (early exponential growth), were further checked for their resistance to erythromycin, a trait of strain Cp1008 (comA: : ery). Seven of 12 clones showed erythromycin resistance and five were erythromycin sensitive; these five were resistant to chloramphenicol, indicating that bacteria from both the Cp1008 comA : : ery $\left(\mathrm{Ery}^{\mathrm{R}}\right)$ and $\mathrm{Cp} 1015$ luxS ${ }^{-}\left(\mathrm{Ery}^{\mathrm{S}}, \mathrm{Cam}^{\mathrm{R}}\right)$ strains were transformed to $\mathrm{Rif}^{\mathrm{R}}$. This indicates that the luxS mutant bacteria produced CSP and activated the Cp1008 subpopulation, and, reciprocally, that late competence of the luxS subpopulation had been repressed by molecules produced by the Cp1008 subpopulation. Such cross-talk between bacteria from both strains accounts for the wildtype-like competence development in the mixed culture. It is likely that deficiency in the CSP-export system ComAB leading to a competence-null phenotype did not abolish the production of signalling molecules deriving from LuxS activity, and that mutation in luxS did not inhibit CSP production. Attempts to inhibit late competence in $5 \mathrm{~h}$ cultures of the luxS mutant strain, using supernatants from the $\operatorname{com} A:$ : ery luxS ${ }^{+}$cultures, showed activity in the media conditioned selectively by $\operatorname{com} A:$ : $\operatorname{ery} \operatorname{lux} S^{+}$and not those conditioned by $\operatorname{com} A$ : : ery lux $S^{-}$bacteria. Late cultures of luxS mutant bacteria were sensitive to conditioned supernatants, and their inhibition level ranged between 20 and $90 \%$ in different experiments (14 independent experiments, data not shown). Taking into account that we were dealing with natural competence development during growth, this variability might be due to the physiological state of each individual $5 \mathrm{~h}$ culture modulating the response to the conditioned supernatant. 


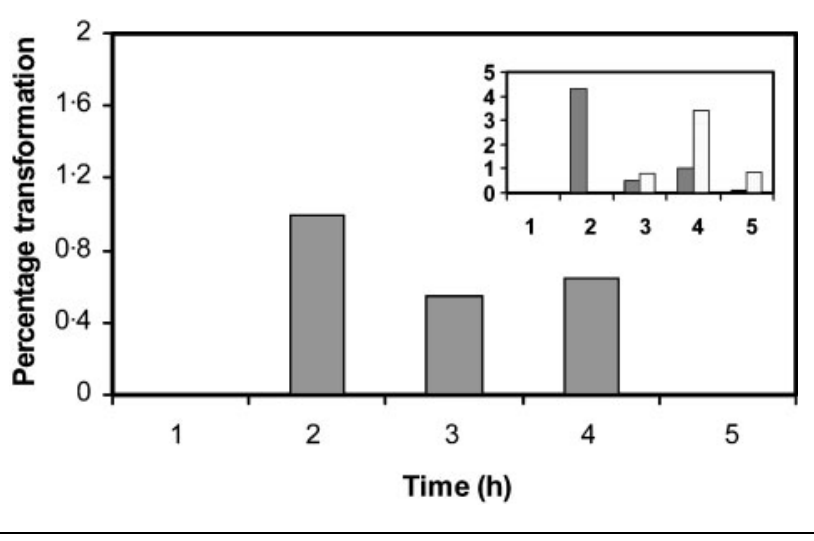

Fig. 3. LuxS mediated cell-cell communication in competence tests. Main graph: mixed cultures (ratio 1:1) of strains Cp1008 (comA::ery) and Cp1015 luxS (lux::cat) grown for 1, 2, 3, 4 and $5 \mathrm{~h}$ were submitted to the competence test (see Methods). The yield of transformant recovery was taken as representative of the competence level of a given culture. In the insert, the profiles of transformant recovery in pure cultures of strain Cp1015 (grey) and Cp1015 luxS (white) are given. The data are representative of three independent experiments.

\section{Impact of LuxS on vegetative growth}

The S-ribosylhomocysteinase activity of LuxS, allowing $S$ adenosyl methionine detoxification and C-1 unit recycling, led us to assess the consequences of LuxS deficiency during vegetative growth. The impact of $l u x S$ mutation on growth appeared dependent on the genetic background and on the $\mathrm{pH}$ of the growth medium. In contrast to neutral cultures $(\mathrm{pH} 7)$, alkaline cultures $(\mathrm{pH} 8)$ of the luxS mutant strain showed accelerated autolysis in late stationary phase, while there was no significant impact on their growth rates (Fig. 4A, B). It has been extensively reported that the muramidase encoded by lytA represents the major autolytic function in S. pneumoniae under these experimental conditions (Lopez et al., 1997; Trombe et al., 1992). LuxSdependent autolysis was indeed suppressed by the lytA:: ery mutation, showing that LuxS activity prevents LytAdependent autolysis and contributes to cellular integrity during stasis (Fig. 4B). Moreover, addition of $0.4 \%$ BSA to the medium (see Methods) protected luxS bacteria from autolysis (Fig. 4C). This was not observed when the BSA concentration was $0.04 \%$ (Fig. 4D), indicating a dosedependent effect of BSA on the integrity of luxS-defective
(A)

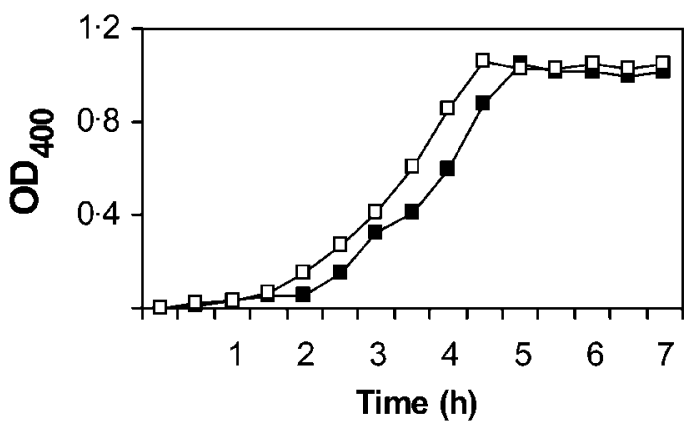

(C)

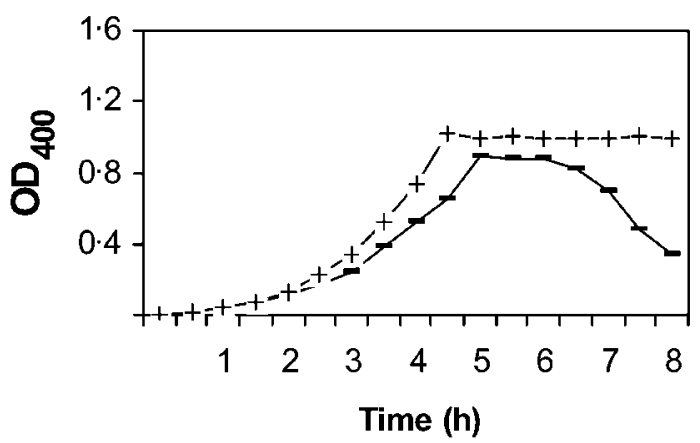

(B)

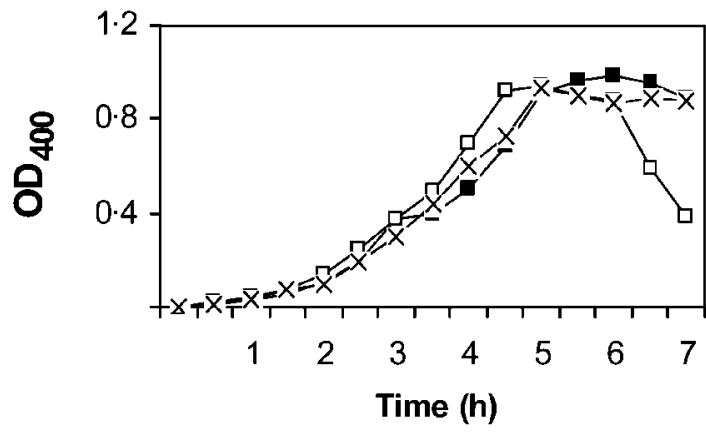

(D)

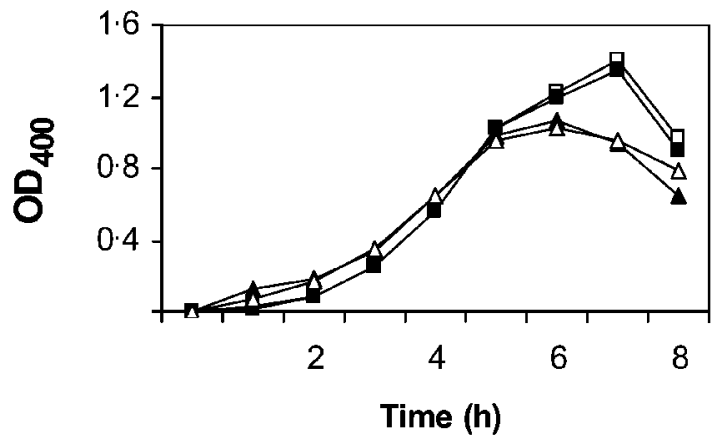

Fig. 4. LuxS involvement in protection against post-exponential LytA-dependent autolysis. (A) Bacteria of lux $S^{+}$(full squares) and $l u x S^{-}$(open squares) strains were grown in CAT medium at $\mathrm{pH} 7$ (A) and $\mathrm{pH} 8$ (B) (Methods) and compared to growth of the luxS lytA strain at $\mathrm{pH} 8$ (crosses, B). (B) Bacteria from the luxS strain were grown at $\mathrm{pH} 8$ in media complemented (crosses) or not complemented (bars) by $0.4 \%$ BSA (C) and by supernatants (10\%) conditioned by Cp1008 (lux $S^{+}$) (open squares) and Cp1008 luxS (closed squares), or 0.04\% BSA (open triangles). The control is shown by closed triangles (D). Growth was monitored by $\mathrm{OD}_{400}$ measurements at $30 \mathrm{~min}$ intervals. The data are representative of at least three independent experiments. 
bacteria during stasis. We never observed (five independent experiments) inhibition of autolysis in $5 \mathrm{~h}$ cultures of the luxS mutant strains by media conditioned by $l u x S^{+}$bacteria, as represented in Fig. 4(D). A consistent increase in growth yield in supplemented cultures was noticed, but it was not related to the nature of the luxS allele, and was not investigated further.

Cellular protection against autolysis during growth in rich medium at alkaline $\mathrm{pH}$ has previously been attributed to StkP and CiaRH. StkP is involved during exponential growth (Authors to be added, unpublished data), and CiaRH is involved during stasis (Guenzy et al. 1994). On the one hand, comparison of the kinetics of autolysis in cultures of strains carrying either the ciaRH or both the ciaRH and the luxS mutations, indicates an additive contribution of LuxS and CiaRH to cellular integrity during stasis (Fig. 5A). On the other hand, the epistasis of the stkP mutation on the
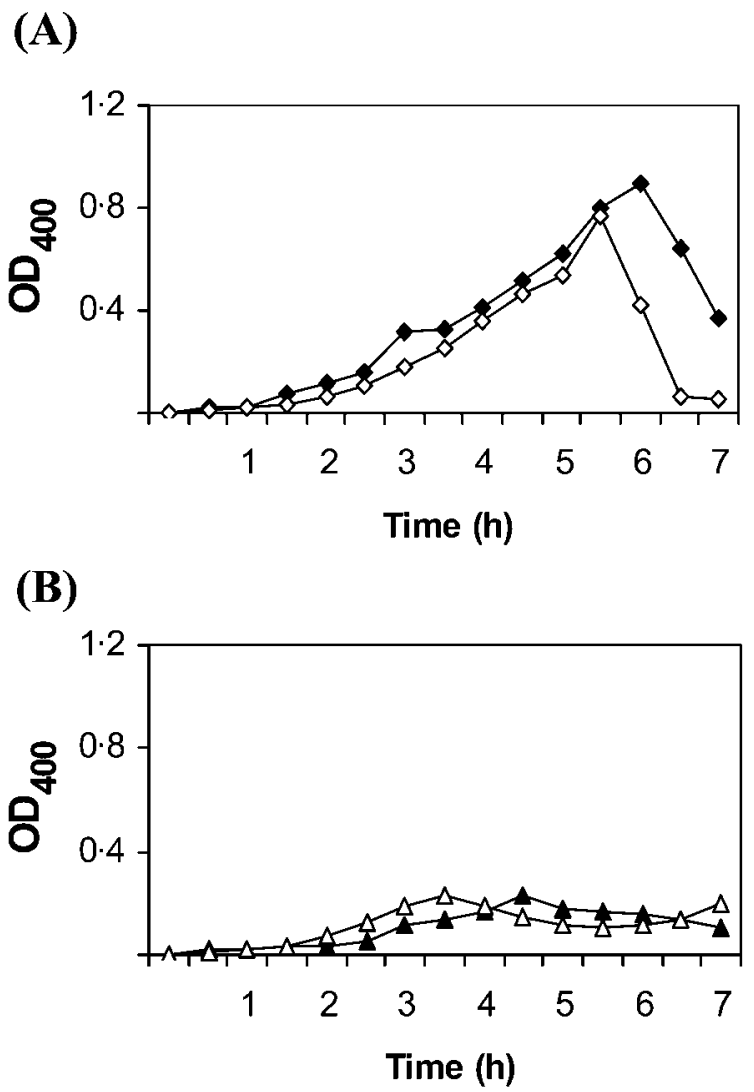

Fig. 5. Relationship between LuxS, StkP and $\mathrm{CiaRH}$ in the control of LytA-dependent autolysis in alkaline cultures. Occurrence of autolysis in cultures in CAT medium at $\mathrm{pH} 8$ (see Methods) of (A) bacteria from strain Cp1800 (ciaR) (closed diamonds) and Cp1800 luxS (ciaR lux) (open diamonds), and (B) bacteria from strain Cp9000 (stkP) (closed triangles) and Cp9000 luxS (stkP luxS) (open triangles). Autolysis was evaluated by the decrease in $\mathrm{OD}_{400}$. The data are representative of three independent experiments.
luxS mutation, illustrated by early autolysis of the luxS stkP mutant strain (Fig. 5B), is consistent with the contribution of StkP and LuxS during exponential growth and stasis, respectively.

\section{DISCUSSION}

In Streptococcus pneumoniae, LuxS appears as a system allowing cell-cell communication, involved in the negative control of competence. LuxS regulation is independent of the ComAB, CSP, ComDE circuit. In addition, LuxS contributes to the control of LytA-dependent autolysis during stationary phase. Autolysis, but not competence, is abolished in media complemented by albumin, suggesting that specific auxotrophy due to LuxS deficiency may account for accelerated autolysis when cultures of $l u x S$ mutant bacteria enter stationary phase.

Competence development in cultures of $S$. pneumoniae shows a net dependence on medium composition, i.e. oxygen concentration, $\mathrm{pH}$ value, and ion and nutrient concentrations. Competence arises specifically in exponentially growing cultures as transient waves, lasting between 30 and 60 min (1-2 doubling times). An interplay of functions involved in intracellular and cell-cell signalling appears to shape the competence waves in pneumococcal cultures. For example, null mutation in the NADH oxidase Nox shifts competence in early exponential-phase cultures and narrows the competence window (Auzat et al., 1999); null mutation in the global regulator RegR further increases the competence defect due to the nox mutation (Chapuy-Regaud et al., 2003). These intracellular regulators act upstream of the cascade of signal transduction involving $\mathrm{CiaRH}$ and ComDE, to trigger CSP production and cell-cell communication (Echenique \& Trombe, 2001). More recently, a novel signalling system consisting of a serine/threonine kinase and its related phosphatase has been characterized (Novakova et al., 2005) and positive regulation of comCDE by the kinase demonstrated (Echenique et al., 2004). Thus, comCDE expression and competence development in growing cultures result from a balance between positive and negative regulation. The relative impact on competence development of a given function, in this complex interplay of positive and negative inputs, may depend on specific growth conditions and genetic backgrounds. Control of LytA-dependent autolysis is also a complex process (Severin et al., 1997; Lopez et al., 1997). Autolysis has classically been described in stationary-phase cultures, and depends on parameters such as the initial $\mathrm{pH}$ of the growth medium and its calcium concentration (Trombe et al. 1992). It is related to competence development (Peterson et al., 2000; Trombe et al. 1992). Recently, LytA-dependent autolysis of exponential cultures has been described in StkP-deficient mutant strains grown at alkaline $\mathrm{pH}$ (M.-C. Trombe \& S. Romao, unpublished data). Therefore, the kinetics of autolysis and competence development represent phenotypes suitable to decipher the regulatory mechanisms that allow cells to adapt to their milieu. Analysis of these phenotypes in vitro and in 
genetically and physiologically defined strains allows a set of possible, putative in vivo regulatory systems to be proposed. The results of genetic dissection in strain Cp1015, a noncapsulated and naturally transformable RX derivative, revealed that LuxS function contributes to cellular integrity during stasis, and participates in intercellular communication in pneumococcal cultures.

\section{Growth and autolysis}

During vegetative growth, LuxS exhibits a protective effect against LytA-dependent autolysis in alkaline cultures entering the stationary phase, a function shared with the TCST system CiaRH. The effect of LuxS is specifically expressed during stasis, and not in exponentially growing cultures. Complementation of the luxS mutation by $0.4 \%$ BSA suggests a metabolic role for LuxS. During the course of this work, a transcriptome analysis was published which suggested that LuxS deficiency culminates in an aberrant transcriptional profile for several genes, including genes involved in lipid biosynthesis (Joyce et al., 2004). Fatty acids are common 'contaminants' of BSA preparations, and may account for BSA complementation of the autolytic phenotype of the luxS mutant bacteria.

\section{Competence development}

The results of the genetic dissection of strain Cp1015, a noncapsulated and naturally transformable RX derivative, revealed the role of LuxS in competence down-regulation via intercellular communication. LuxS regulation appears to be independent of the CSP, ComAB, ComDE regulatory network. The TCST system CiaRH is the major negative regulator of competence (Guenzy et al., 1994), and controls the cellular level of comCDE mRNA (Echenique et al., 2000). In a competence-derepressed ciaRH mutant strain, luxS mutation further increases the competence level and the time-window of expression, suggesting additive competence repression by LuxS and CiaRH. StkP increases the cellular level of comCDE mRNA (Echenique et al., 2004); suppression of competence deficiency due to $s t k P$ mutation by the luxS mutation reveals other positive inputs triggering competence in late cultures.

The results of mixed-culture experiments allow us to propose that LuxS activity culminates in the production of 'molecules' active towards competence development. The production of these factors is independent of the $A B C$ transporter ComAB, because in mixed cultures, $\operatorname{comA}:$ : ery luxS $S^{+}$bacteria impact on the competence profile of $l u x S^{-}$ bacteria. Variability in the response of luxS mutant cell cultures to media conditioned by $\operatorname{luxS} S^{+}$bacteria might be due to the 'late competence phenotype', which may represent a vanishing process difficult to control. In any case, our data indicate that media conditioned by $l u x S^{+}$bacteria are active towards $\operatorname{luxS} S^{-}$bacteria. We have not tested these supernatants in the $V$. harveyi model. In independent investigations, supernatants from strain D39 grown in BHI have given opposite results to those of this test (Stroeher et al., 2003; Joyce et al., 2004). It is possible that growth conditions or genetic variation between isolates of strain D39 account for these discrepancies. More clear-cut phenotypes and genetic backgrounds have to be elaborated to draw conclusions about AI-2 production due to LuxS activity in S. pneumoniae.

Currently, the production by S. pneumoniae of AI-2 active towards $V$. harveyi is uncertain (Stroeher et al., 2003; Joyce et al., 2004). Indeed, the metabolic pathway that allows furanone production involves different enzymes, although corresponding genes are present in the pneumococcal genome. The metabolic pathway contains steps catalysed by an $S$-adenosylmethionine synthetase (most probable Kyoto Encyclopedia of Genes and Genomes, KEGG, annotation for S. pneumoniae is SP0762; http://www.genome.ad.jp/kegg/), a methyltransferase (most probable pneumococcal gene is SP1336), an S-adenosylhomocysteine nucleosidase (most probable pneumococcal gene is SP0991) and LuxS (SP0340). However, neither the luxP and luxQ orthologues encoding the signal transduction system targeted by furanones, nor the $l s r$ operon allowing uptake and processing of AI-2 in Gram-negative bacteria, have been identified in the pneumococcal genome. The actors in cell-cell communication dependent on LuxS function remain to be characterized.

In conclusion, the data presented provide credence for pleiotropic consequences of the loss of S-ribosylhomocysteinase, LuxS, function in Streptococcus pneumoniae in vitro. It is suggested that LuxS is involved in the intracellular control of LytA-dependent autolysis, as well as in cell-cell communication culminating in competence repression. Indeed, studies in the Gram-positive Streptococcus gordoni have allowed the proposal that AI-2 production is regulated at the metabolic level (Blehert et al., 2003). In Salmonella enterica serovar Typhimurium, AI-2 production depends on the level of LuxS substrate availability, rather than luxS expression, and reflects the metabolic state of the cell (Beeston \& Surette, 2002). In S. pneumoniae, LuxS may contribute to the amplification of cellular metabolic signals to the whole population via the production of molecules allowing cell-cell communication.

In the wild-type genetic background, the role of LuxS in competence and autolysis is modest, likely due to the preeminent impact of StkP and CiaRH on the traits analysed. This indicates a hierarchy in competence signalling in vitro, with a minor role for LuxS. It cannot be excluded that LuxS may play a more important role under other environmental conditions.

\section{ACKNOWLEDGEMENTS}

The work in Sienna was financed by a grant from Ministero dell'Istruzione, dell'Università e della Ricerca (MIUR, Italian Ministry of Research) (FIRB RBAU01X9TB) to M. R. O. The work in Toulouse was financed by Université Paul Sabatier, Toulouse. S. R. was on a European ERASMUS exchange programme. We thank Saliha Mimar for technical help. 


\section{REFERENCES}

Auzat, I., Chapuy-Regaud, S., Dos Santos, D., Le Thomas, I., Le Bras, G., Onnuyighy, D., Garel, J.-R., Paton, J. \& Trombe, M.-C. (1999). The NADH oxidase of Steptococcus pneumoniae, its role in competence and virulence. Mol Microbiol 34, 1018-1028.

Bassler, B. L., Greenberg, E. P. \& Stevens, A. M. (1997). Crossspecies induction of luminescence in the quorum sensing bacterium Vibrio harveyi. J Bacteriol 179, 4043-4045.

Beeston, A. L. \& Surette, M. G. (2002). pfs-dependent regulation of autoinducer-2 production in Salmonella enterica serovar Typhimurium. J Bacteriol 184, 3450-3456.

Blehert, D. S., Palmer, R. J., Jr, Xavier, J. B., Almeida, J. S. \& Kolenbrander, P. E. (2003). Autoinducer 2 production by Streptococcus gordonii DL1 and the biofilm phenotype of a luxS mutant are influenced by nutritional conditions. J Bacteriol 185, 4851-4860.

Chapuy-Regaud, S., Ogunniyi, D., Diallo, N., Huet, Y., Desnottes, J.-F., Paton, J., Escaich, S. \& Trombe, M.-C. (2003). RegR, a global LacI/GalR family regulator, modulates virulence and competence in Streptococcus pneumoniae. Infect Immun 71, 2615-2625.

Chen, X., Schauder, S., Potier, N., Van Dorsselaer, A., Pelczer, I., Bassler, B. L. \& Hughson, F. M. (2002). Structural identification of a bacterial quorum-sensing signal containing boron. Nature 415, 545-549.

Claverys, J. P., Dintilhac, A., Pestova, E. V., Martin, B. \& Morrison, D. A. (1995). Construction and evaluation of new drug-resistance cassettes for gene disruption mutagenesis in Streptococcus pneumoniae, using an ami test platform. Gene 164, 123-128.

Dove, J. E., Yasukawa, K., Tinsley, C. R. \& Nassif, X. (2003). Producion of the signaling molecule autoinducer-2 by Neisseria meningitis: lack of evidence for a concerted transcription response. Microbiology 149, 1859-1869.

Echenique, J. \& Trombe, M.-C. (2001). Competence modulation by Nox involves signal transduction in Streptococcus pneumoniae. $J$ Bacteriol 183, 768-772.

Echenique, J., Chapuy-Regaud, S. \& Trombe, M.-C. (2000). Oxygen regulation of competence inStreptococcus pneumoniae: involvement of ciaRH and comcDE. Mol Microbiol 36, 688-696.

Echenique, J., Kadioglu, A., Romao, S., Andrew, P. W. \& Trombe, M.-C. (2004). The protein serine/theonine kinase, StkP, positively controls virulence and competence in Streptococcus pneumoniae. Infect Immun 72, 2434-2437.

Guenzy, E., Gasc, A. M., Sicard, M. A. \& Hakenbeck, R. (1994). A two component signal-transducing system is involved in competence and penicillin susceptibility in laboratory mutants of Streptococcus pneumoniae. Mol Microbiol 12, 505-515.

Hammer, B. K. \& Bassler, B. L. (2003). Quorum sensing controls biofilm formation in Vibrio cholerae. Mol Microbiol 50, 101-104.

Henke, J. M. \& Bassler, B. L. (2004). Three parallel quorum sensing systems regulate gene expression in Vibrio harveyi. J Bacteriol 186, 6902-6914.

lannelli, F. \& Pozzi, G. (2003). Method for introducing specific and unmarked mutations into the chromosome of Streptococcus pneumoniae. Mol Biotechnol 26, 81-86.

Joyce, E. A., Kawale, A., Censini, S., Kim, C. C., Covacci, A. \& Falkow, S. (2004). LuxS is required for persistent pneumococcal carriage and expression of virulence and biosynthesis genes. Infect Immun 72, 2964-2975.

Kwon, H. Y., Kim, S. W., Choi, M. H., Ogunniyi, A. D., Paton, J. C., Park, S. H., Pyo, S. N. \& Rhee, D. K. (2003). Effect of heat shock and mutations in $C l p L$ and $C l p P$ on virulence gene expression in Streptococcus pneumoniae. Infect Immun 71, 3757-3765.

Lopez, R., Garcia, E., Garcia, P. \& Garcia, J. L. (1997). The pneumococcal cell wall degrading enzymes: a modular design to create new lysins. Microb Drug Resist 3, 199-211.

Maroui, M. J. \& Sela, S. (2003). The luxS gene of Streptococcus pyogenes regulates expression of genes that affect internalization by epithelial cells. Infect Immun 71, 5633-5639.

Massidda, O., Anderluzzi, D., Friedli, L. \& Feger, G. (1998). Unconventional organization of the division and cell wall gene cluster of Streptococcus pneumoniae. Microbiology 144, 3069-3078.

Miller, M. B., Skorupski, K., Lenz, D. H., Taylor, R. K. \& Bassler, B. L. (2002). Parallel quorum sensing systems converge to regulate virulence in Vibrio cholerae. Cell 110, 303-304.

Morrison, D. A. \& Lee, M. S. (2000). Regulation of competence for genetic transformation in Streptococcus pneumoniae: a link between quorum sensing and DNA processing genes. Res Microbiol 151, 445-451.

Morrison, D., Trombe, M.-C., Hayden, G., Waszack, G. \& Chen, J.-D. (1984). Isolation of transformation deficient mutants of Steptococcus pneumoniae defective in control of competence, using insertionduplication mutagenesis with EM determinant of pAMB1. J Bacteriol 159, 870-876.

Novakova, L., Saskova, L., Pallova, P., Janecek, J., Novotna, J., Ulrych, A., Echenique, J., Trombe, M. C. \& Branny, P. (2005). Characterisation of a eukaryotic type serine/threonine protein kinase and protein phosphatase of Streptococcus pneumoniae and identification of kinase substrates. FEBS J 272, 1243-1254.

Palmen, R., Ogunniyi, D., Berroy, P., Larpin, S., Paton, J. \& Trombe, M.-C. (1999). Insertional mutation of orfD of the DCW cluster of Streptococcus pneumoniae attenuates virulence. Microb Pathog 27, 337-348.

Peterson, S., Cline, R. T., Tettelin, H., Sharov, V. \& Morrison, D. A. (2000). Gene expression analysis of the Streptococcus pneumoniae competence regulon by use of DNA microarrays. J Bacteriol 182, 6192-6202.

Robertson, G. T., Ng, W. L., Foley, J., Gilmour, R. \& Winkler, M. E. (2002). Global transcriptional analysis of clpP mutations of type 2 Streptococcus pneumoniae and their effects on physiology and virulence. J Bacteriol 184, 3508-3520.

Schauder, S., Penna, L., Ritton, A., Manin, C., Parker, F. \& RenaultMongenie, G. (2005). Proteomic analysis by two-dimensional differential gel electrophoresis reveals the lack of a broad response of Neisseria meningitis to in vitro-produced AI-2. J Bacteriol 187, 392-395.

Severin, A., Horne, D. \& Tomasz, A. (1997). Autolysis and cell wall degradation in a choline-independent strain of Streptococcus pneumoniae. Microb Drug Resist 3, 391-400.

Sperandino, V., Torres, A. G., Jarvis, B., Nataro, J. P. \& Kaper, J. B. (2003). Bacteria-host communication: the language of hormones. Proc Natl Acad Sci U S A 100, 8951-8956.

Stroeher, U. H., Paton, A. W., Ogunniyi, A. D. \& Paton, J. C. (2003). Mutation of luxS of Streptococcus pneumoniae affects virulence in a mouse model. Infect Immun 71, 3206-3212.

Sun, J., Daniel, R., Wagner-Doble, R. I. \& Zeng, A. P. (2004). Is autoinducer-2 a universal signal for interspecies communication: comparative genomic and phylogenetic analysis of the synthesis and transduction pathways. BMC Evol Biol 4, 36-47.

Surette, M. G., Miller, M. B. \& Bassler, B. L. (1999). Quorum sensing in Escherichia coli, Salmonella typhimurium and Vibrio harveyi: a new family of genes responsible for autoinducer production. Proc Natl Acad Sci U S A 96, 1639-1644. 
Taga, M. E. \& Bassler, B. L. (2003). Chemical communication among bacteria. Proc Natl Acad Sci U S A 100, 14549-14554.

Taga, M. E., Miller, S. T. \& Bassler, B. L. (2003). Lsr-mediated transport and processing of AI-2 in Salmonella typhimurium. Mol Microbiol 50, 1411-1427.

Trombe, M.-C., Clavé, C. \& Manias, J. M. (1992). Calcium regulation of growth and differentiation in Streptococcus pneumoniae. J Gen Microbiol 139, 433-439.
Vendeville, A., Winzer, K., Heurlier, K., Tang, C. M. \& Hardie, K. R. (2005). Making 'sense' of metabolism: autoinducer-2, LuxS and pathogenic bacteria. Nat Rev Microbiol 3, 383-396.

Xavier, K. B. \& Bassler, B. L. (2003). LuxS quorum sensing: more than just a numbers game. Curr Opin Microbiol 6, 191-197.

Xavier, K. B. \& Bassler, B. L. (2005). Regulation of uptake and processing of the quorum-sensing autoinducer AI-2 in Escherichia coli. J Bacteriol 178, 238-248. 